\title{
Automated methods for improved characterization of alloy nanoparticle catalysts
}

David Cullen ${ }^{1}$, Michael Zachman ${ }^{1}$, Haoran $\mathrm{Yu}^{2}$, Debangshu Mukherjee ${ }^{3}$ and Shawn Reeves ${ }^{4}$

${ }^{1}$ Center for Nanophase Materials Sciences, Oak Ridge National Laboratory, Tennessee, United States, ${ }^{2}$ Center for Nanophase Materials Sciences, Oak Ridge National Laboratory, Oak Ridge, Tennessee, United States, ${ }^{3}$ Center for Nanophase Materials Sciences, Oak Ridge National Laboratory, KNOXVILLE, Tennessee, United States, ${ }^{4}$ ORNL, United States

Supported metallic nanoparticles are an important class of catalysts which are utilized in a wide range of applications [1]. In many cases, understanding degradation mechanisms is critical for enabling more robust operation. For example, heavy duty vehicles are an emerging application for polymer electrolyte membrane fuel cells, which rely on a Pt-based nanoparticle catalysts at both the anode and cathode [2,3]. Such vehicles can log more than one million miles over their lifetime, and the durability of the supported catalysts during this extended operation is considered a critical barrier to commercialization [4]. Accelerated stress tests (ASTs) have been developed to elucidate degradation mechanisms, but generate large sample series which call for rapid development of high throughput, statistically relevant techniques for measuring changes in particle size and composition.

In this work, we employ automated data acquisition and analysis methods for increased throughput and sampling size in the electron microscope. As shown in Figure 1, the Thermo Scientific Maps Software was used to automatically acquire high-angle annular dark-field scanning transmission electron microscopy (HAADF-STEM) images for particle size measurements across the fuel cell electrodes. The thousands of particles and dozens of spectrum images taken during an automated overnight run present a significant challenge for data analysis. Custom Python codes are being optimized for particle diameter measurements, including advanced methods of treating particle overlap common in these supported catalysts. We will also introduce new methods for quantifying the dispersion of the proton-conducting ionomer within the electrode, a metric which until now has only been qualitatively assessed.

Alloy nanoparticles offer many potential advantages over their monometallic counterparts. In the case of fuel cell catalysts, strain induced on a thin Pt-skin by the underlying PtCo alloy core can significantly enhance mass activity towards the oxygen reduction reaction [2]. However, such core-shell structures are unstable in the acidic fuel cell environment, leading to leaching of the Co core and growth of the Pt skin during operation. As shown in Figure 2, methods for rapidly measuring changes in Pt-skin thickness using radial averaging methods have been developed. Combined with large solid angle energy dispersive X-ray spectrometers (EDS), dozens of particles can be measured to show how degradation varies by particle size, location within the porous support, and proximity to the fuel cell inlet and outlet. In this work, we will also compare conventional homogenous core catalysts to emerging classes of intermetallic nanoparticles to elucidate differences in degradation which may improve the durability and stability of these highly active materials.

More specialized techniques like 4D-STEM and electron tomography also play an important role in understanding the effect of surface strain and catalyst-support interactions on activity and durability. The outlook for increasing the sampling size for 4D-STEM datasets, accelerating tomography reconstruction with machine learning, and automating segmentation of 3D datasets for these classes of supported nanoparticles will also be discussed.

Research sponsored by the Hydrogen and Fuel Cell Technologies Office, Office of Energy Efficiency and Renewable Energy, US Department of Energy (DOE). Research was conducted at the Center for Nanophase 
Materials Sciences, which is a DOE Office of Science User Facility. The Talos F200X S/TEM tool provided by US DOE, Office of Nuclear Energy, Fuel Cycle R\&D Program and the Nuclear Science User Facilities.
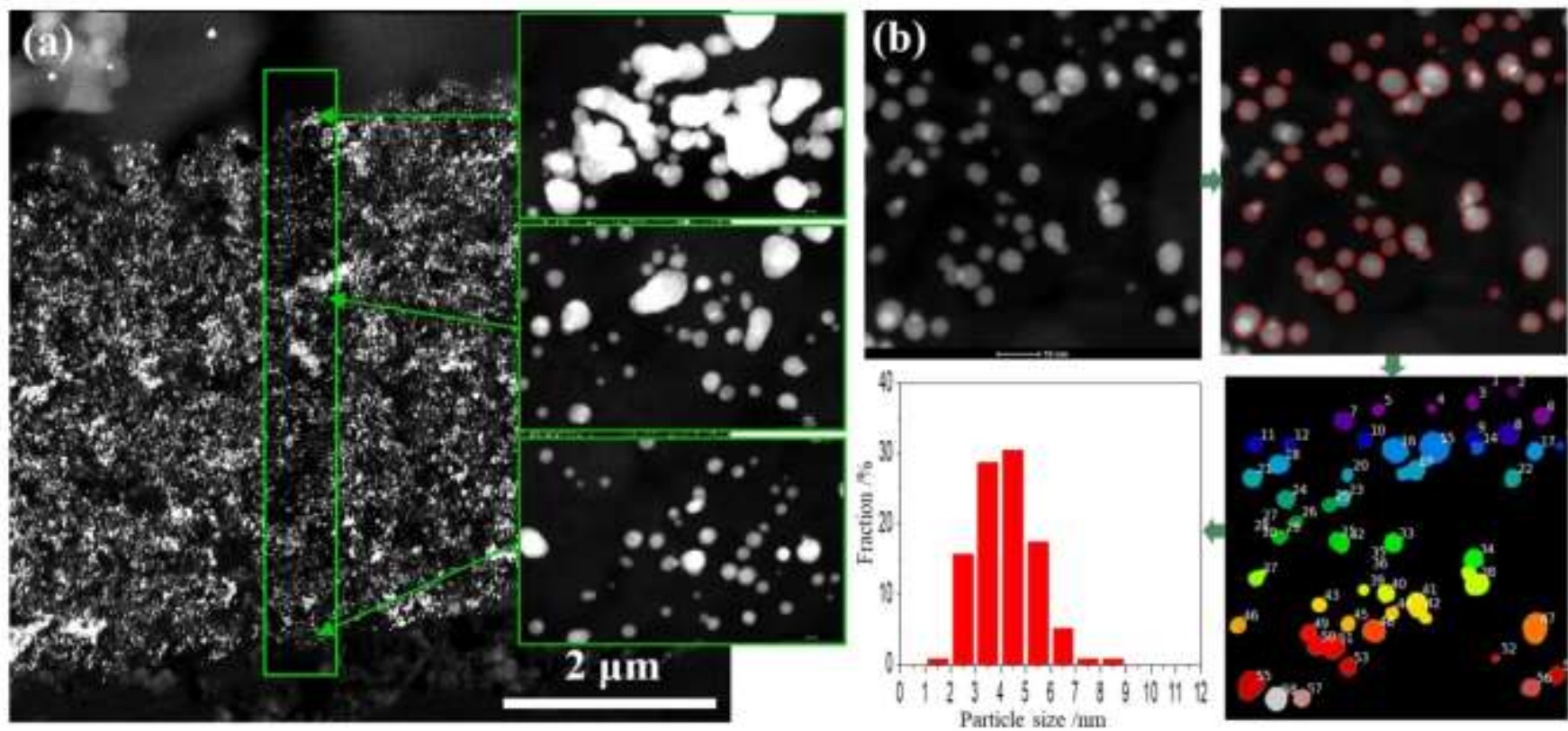

Figure 1. Figure 1. (a) Example HAADF-STEM images of PtCo nanoparticles from different regions of a fuel cell cathode captured using the automated image acquisition feature in the Thermo Scientific Maps software and (b) high-throughput particle size measurements using custom python codes.
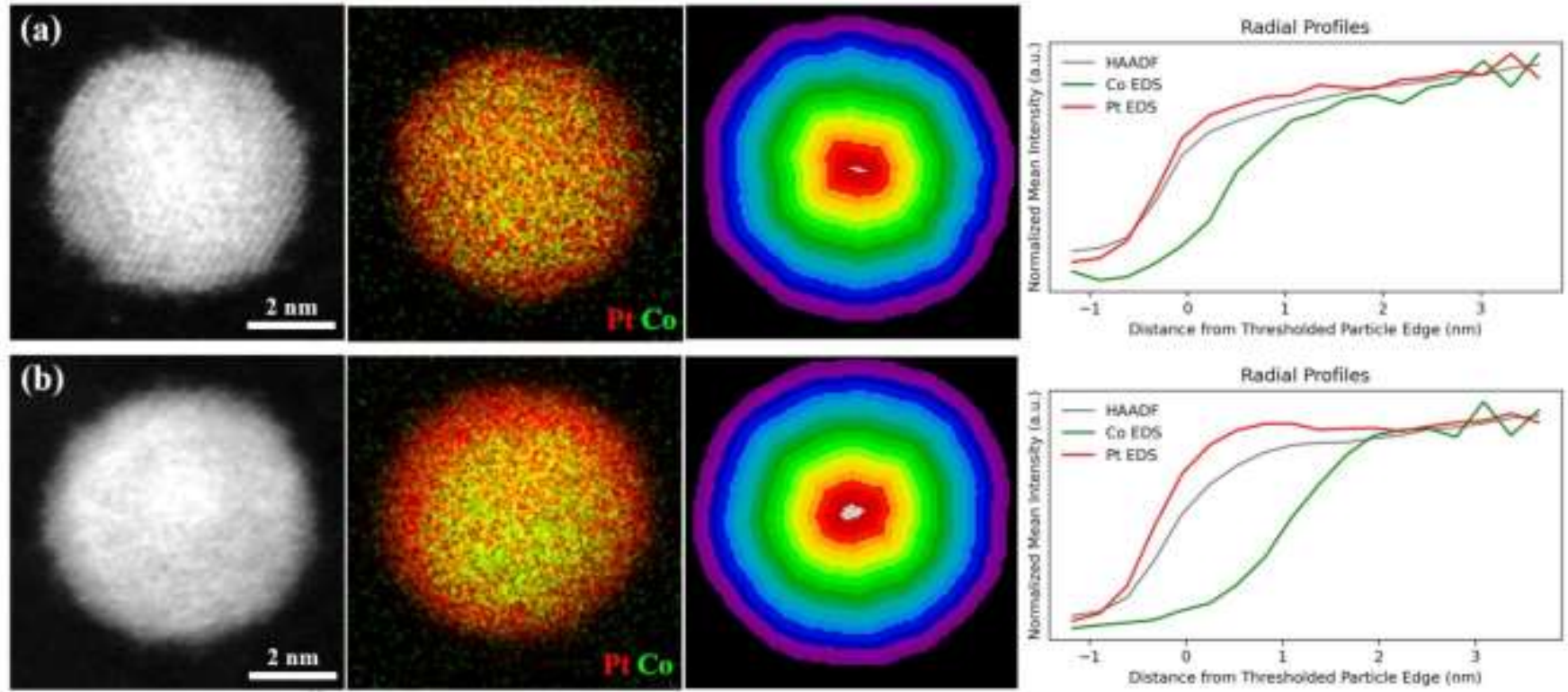

Figure 2. Figure 2. High resolution HAADF-STEM and EDS spectrum images for Pt (red) and Co (green) with radial averaged profiles (a) before and (b) after accelerated stress tests showing the change in the Ptshell thickness on the PtCo core. In the third panel, each color displayed indicates a separate region where EDS spectra were summed to produce the points in the profiles shown on the right. 


\section{References}

[1] D. Astruc, Chem. Rev. 120 (2020), p. 461.

[2] XX Wang, MT Swihart, and G Wu, Nat. Catal. 2 (2019), p. 578.

[3] RL Borup et al., Curr. Opin. Electrochem. 21 (2020), p. 192.

[4] J. Marcinkoski et al., Electrified Powertrain Roadmap (2019), 19006. 